\title{
Prediction of extreme geophysical, industrial and biophysical flows using particle methods
}

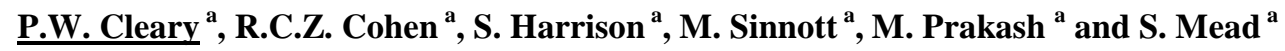 \\ ${ }^{a}$ Mathematics, Informatics and Statistics, CSIRO, Clayton, Victoria \\ Email: Paul.Cleary@csiro.au
}

\begin{abstract}
The flow of particulates and fluids is critical to many applications. Examples include human processes such as eating and locomotion, extreme geophysical flows such as tsunamis and dam-collapse inundation, biomedical applications such as transport of material in the gastro-intestinal tract and heart assist pumps, and industrial processes such as crushing and grinding (comminution).
\end{abstract}

Meshless particle based methods such DEM (Discrete Element Method) and SPH (Smoothed Particle Hydrodynamics) have a range of strong advantages over traditional grid based continuum methods for these types of applications. The advantages include:

- the ability to include particle level information in collision dominated particle flows

- $\quad$ ability to resolve complex fluid free surfaces including splashing and fragmentation

- $\quad$ ability to predict very large deformations, including fracture

- $\quad$ interaction with moving and deforming solid bodies or objects

- ability to track material history and use this in the flow modelling such as the rheology.

These advantages are demonstrated in a series of computationally demanding examples and case studies including crushers and mills, biomedical pumps and transport of faecal matter in the bowel, geophysical extreme flow events (risk/disaster modelling), eating of food by humans and elite water based sports.

In all cases, there are significant sources of variability that need to be taken into account in the modelling:

- For human based models the major sources of variability are the nature of the motions of the human (both internal and external). Such variations can be short term (cycle variation between repetitions), medium term such as changes in response to fatigue or the relative timing and strengths of repetitions over time or longer term (such as in response to injury or deliberate planned changes).

- For the geophysical/disaster modelling, variability is mainly reflected in the range and nature of the scenarios that need to be considered in building risk frameworks in which these models can predict specific consequences. For examples, the range of possible failure scenarios of a dam or the range of heights, speeds and water volumes for tsunamis generated by poorly understood initiation events.

- Finally, in all such applications there is uncertainty about material properties and the details of the initial conditions whose effects need to be understood. For example, in comminution (either in an industrial crusher or mill or in the human mouth during chewing) the performance of the process is heavily influenced by the specific nature of the material being comminuted. This can vary strongly between materials and between different instances of what is nominally the same material. In the human eating case, this is further complicated by feedback from the material nature into the detailed nature of the chewing motion.

Such variability influences both the inputs to specific instances of the deterministic models and the usage scenarios that need to be considered to understand the range of behaviours that exist for these processes. Optimisation and decision making based on such models cannot be reasonably performed without considering these issues. The ultimate usefulness of the model outcomes is heavily dependent on the quality of the management of these uncertainties.

Keywords: Smoothed particle hydrodynamics (SPH), discrete element method (DEM), geophysical flows, industrial flows, biophysical flows 


\section{INTRODUCTION}

DEM has been developed and used over the last 30 years for modelling flows of particulate solids in many applications, starting with small systems in simple geometries in two-dimensions (Cundall and Strack, 1979; Walton, 1994; Campbell, 1990; Haff and Werner, 1986). It is now possible to simulate systems of tens of millions of particles on desktop computers (Cleary, 2004, 2009) enabling many complex particulate flows to be predicted. It is the most effective method for modelling flow controlled by collisions of particulates.

SPH is a powerful particle method well suitable for solving complex multi-physics flow and deformation problems. It is particularly well suited to splashing free surface flows, interaction with dynamic moving bodies and discrete particles. It also has strong advantages in situations where flow or material history is important. The method was first developed for use with incompressible fluid flows by Monaghan (1994). Many examples of the application of SPH are given in Cleary et al. (2007).

The inherent flexibility of these two Lagrangian techniques allows them to be easily and effectively applied to a wide range of different modelling problems. An important issue for such modelling is the inherent uncertainties that surround it. Whilst each simulation is deterministic in nature, there are often uncertainties in model inputs and in the range of scenarios that can occur with each flow. In this paper we describe a subset of the applications where such numerical methods have been applied and highlight the diversity of physics and modelling scenarios that can be readily handled by DEM and SPH and how uncertainty influences such modelling.

\section{PARTICLE METHODS}

DEM is a simulation method that models particulate systems whose motions are dominated by collisions. This involves following the motion of every particle or object in the flow and modelling each collision between the particles and between the particles and their environment. The algorithm has three main stages:

1. A search grid is used to periodically build a near-neighbour interaction list that contains all the particle pairs and object-particle pairs that are likely to experience collisions in the short term.

2. The forces on each pair of colliding particles and/or boundary objects are evaluated in their local reference frame using a suitable contact force model, and then transformed into the simulation.

3. All the forces and torques on each particle and object are summed and the resulting equations of motion are integrated to give the resulting motion of these bodies.

The implementation used here is described in more detail in Cleary $(2004,2009)$. The entities are allowed to overlap and the amount of overlap $\Delta x$, and normal $v_{n}$ and tangential $v_{t}$ relative velocities determine the collisional forces via a contact force law. We use a linear spring-dashpot model where the normal and tangential forces are given by:

$$
F_{n}=-k_{n} \Delta x+C_{n} v_{n}, \quad F_{t}=\min \left\{\mu F_{n}, k_{t} \int v_{t} d t+C_{t} v_{t}\right\}
$$

The normal force consists of a linear spring to provide the repulsive force and a dashpot to dissipate a proportion of the relative kinetic energy. The maximum overlap between particles is determined by the stiffness $k_{n}$ of the spring in the normal direction. Typically, average overlaps of $<0.5 \%$ of the particle diameter are desirable. The normal damping coefficient $C_{n}$ is chosen to give the required coefficient of restitution $\varepsilon$. The vector force $F_{t}$ and velocity $v_{t}$ are defined in the plane tangent to the surface at the contact point. The tangential integral term represents an incremental spring that stores energy from the relative tangential motion and models the elastic tangential deformation of the contacting surfaces, while the dashpot dissipates energy and models tangential plastic. The total tangential force $F_{t}$ is limited by the Coulomb frictional limit $\mu F_{n}$, at which point the surface contact shears and the particles begin to slide over each other.

The SPH method uses a meshless spatial discretisation to convert systems of PDEs into coupled systems of ODEs that can then be solved using suitable time integration methods. For fluids, the continuity equation is

$\frac{d \rho_{a}}{d t}=\sum_{b} m_{b}\left(\mathbf{v}_{a}-\mathbf{v}_{b}\right) \bullet \nabla W_{a b}$

where $\rho_{a}$ is the density of particle $a$ with velocity $\mathbf{v}_{a}$ and $m_{b}$ is the mass of particle $b$. We denote the position vector from particle $b$ to particle $a$ by $\mathbf{r}_{a b}=\mathbf{r}_{a}-\mathbf{r}_{b}$ and the velocity difference by $\mathbf{v}_{a b}=\mathbf{v}_{a}-\mathbf{v}_{b}$. $W_{a b}=W\left(\mathbf{r}_{a b}, h\right)$ is the interpolation kernel with smoothing length $h$ evaluated at distance $\left|\mathbf{r}_{a b}\right|$. The SPH method used here is quasi-compressible formulation with an equation of state specifying the relationship between particle density and fluid pressure. A form suitable for incompressible fluids is: 


$$
P=P_{0}\left\lfloor\left(\frac{\rho}{\rho_{0}}\right)^{\gamma}-1\right\rfloor
$$

where $P_{0}$ is the magnitude of the pressure given by

$$
\frac{g P_{0}}{r_{0}}=100 \mathrm{~V}^{2}=c^{2}
$$

and $V$ is the characteristic or maximum fluid velocity and $c$ is the speed of sound. This means that the sound speed is ten times the characteristic speed and ensures that the density variation is less than $1 \%$ and that the flow is close to incompressible. $\rho_{0}$ is the reference density and $\gamma=7$.

The SPH form of the momentum equation becomes the acceleration for each particle $a$ :

$$
\frac{d \mathbf{v}_{a}}{d t}=\mathbf{g}-\sum_{b} m_{b}\left\lfloor\left(\frac{P_{b}}{\rho_{b}^{2}}+\frac{P_{a}}{\rho_{a}^{2}}\right)-\frac{\xi}{\rho_{a} \rho_{b}} \frac{4 \mu_{a} \mu_{b}}{\left(\mu_{a}+\mu_{b}\right)} \frac{\mathbf{v}_{a b} \mathbf{r}_{a b}}{\mathbf{r}_{a b}^{2}+\eta^{2}}\right\rfloor \nabla_{a} W_{a b}
$$

where $P_{a}$ and $\mu_{a}$ are pressure and viscosity of particle $a$. Here $\xi$ is a factor associated with the viscous term, $\eta$ is a small parameter used to smooth out the singularity at $\mathbf{r}_{a b}=0$ and $\boldsymbol{g}$ is gravity.

\section{INDUSTRIAL COMMINUTION}

Comminution is the process of reducing the size of particles by breakage. It starts with large particles which are typically broken by one or more stages of crushing. The intermediate size particles produced are then fed to grinding mills that grind the particles down to $\mathrm{mm}$ or micron size. DEM simulations of a crusher and a mill are presented. Figure 2a shows a DEM model of a cone crusher (around $0.6 \mathrm{~m}$ wide and $0.4 \mathrm{~m} \mathrm{high}$ ). The crusher is choke fed from above with medium size material $(10-40 \mathrm{~mm})$. The mantle (the moving conical section in the middle) is inclined at $1^{\circ}$ and moves with a nutating motion at $600 \mathrm{rpm}$. The concave (the outer object) is stationary. At any circumferential location the mantle oscillates away from and then toward the concave causing particles to fall into the crushing zone and be compressed and fractured. The DEM model uses dynamic breakage of the particles (with a compression breakage rule). This permits the coarse parent particles to break and the daughter particles to move lower in the crusher and be re-broken before exiting the crusher at the bottom. This allows prediction of key machine outputs, including the power draw which was $9.5 \mathrm{~kW}$ and the throughput of 11.5 tonnes/hr. The flow pattern in the crusher is very reproducible, but the prediction of throughput and the product size distribution is very dependent on the variability of the material properties of the feed material. Softer or heavily flawed rock breaks much more easily than competent ore.

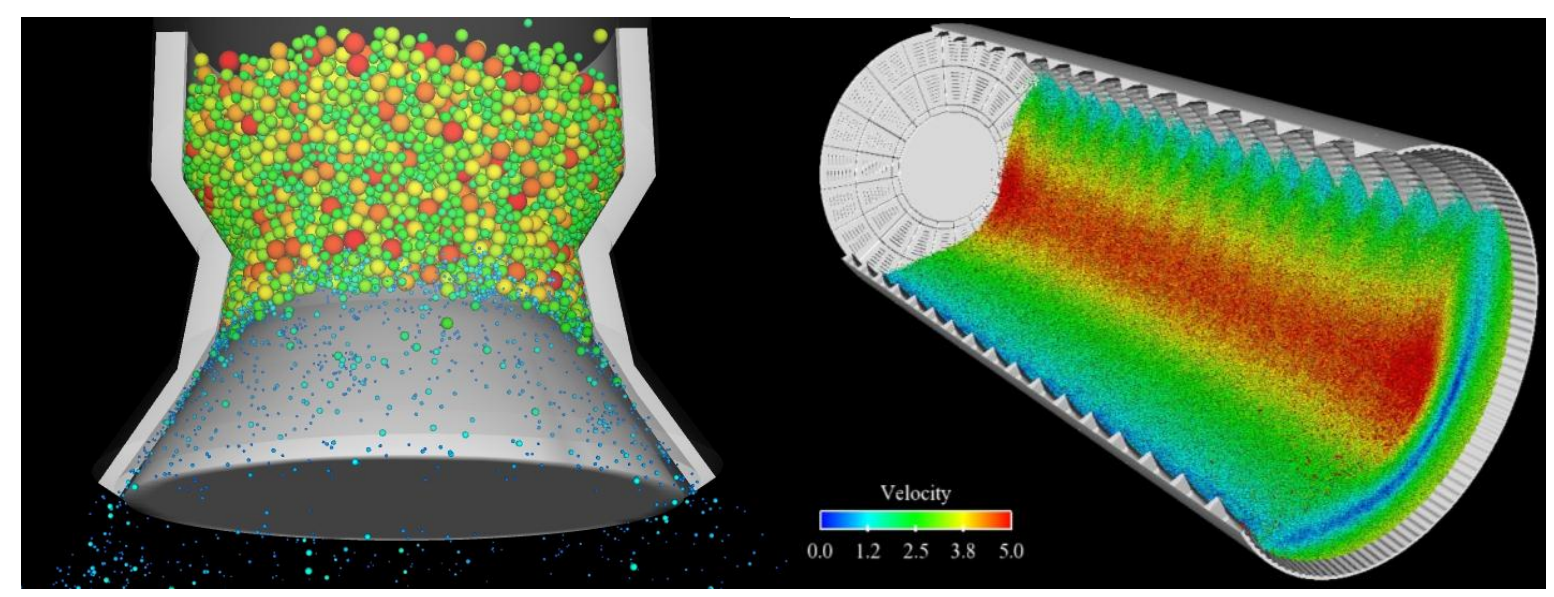

Figure 1. a) Particle distribution in a cone crusher with particles coloured by size (red are large, green intermediate and blue are small), and b) flow pattern of grinding balls in the second chamber of a cement mill. The particles are coloured by velocity with red being fast $(5 \mathrm{~m} / \mathrm{s})$ and blue being slow $(<1 \mathrm{~m} / \mathrm{s})$.

Grinding of clinker for cement production is often performed in a two chamber ball mill. In the first shorter chamber, raw clinker feed is ground with the product being transferred to the second longer chamber. Here smaller balls are used to grind the product material even finer. We model such a second chamber of a cement ball mill. It has an inner diameter of $3.85 \mathrm{~m}$, is $8.4 \mathrm{~m}$ long and rotates at $16.13 \mathrm{rpm}$ (75\% critical speed). It 
has a classifying liner with a symmetric wave profile and 120 wave peaks around the circumference of the mill. The feed end of each lifter has a vertical step up to its highest point and the height then decreases along the lifter. There are 17 sets of these lifters along the axis of the mill. The ball size ranges from 15 to $50 \mathrm{~mm}$ and the fill level is $30 \%$ by volume, leading to a ball charge of 136.4 tonnes consisting of 3.2 million balls.

Figure $2 \mathrm{~b}$ shows the charge motion in the mill with particles being dragged around by the mill shell to a shoulder position where they become mobile and flow down as a cascading stream to the toe position. They are then re-captured by the liner and begin to circulate again. The free surface has the characteristic $\mathrm{S}$ shape. The particles move slowly near the shoulder and toe. As they flow down the surface they accelerate reaching peak speeds of $5 \mathrm{~m} / \mathrm{s}$ in the steep central section. The particles near the mill shell are transported around with the mill rotation at speeds of close to $3 \mathrm{~m} / \mathrm{s}$. There is a narrow band of dark blue connecting the shoulder and toe. This material is very slow moving. Between the slow moving semi-circular blue band and the mill shell is a region of strong shear. Similarly, there is high shear between the slow moving layer and the high speed cascading flow above. Fine clinker particles are systematically trapped and crushed between the balls as they pass each other in these separate sliding layers.

\section{EATING}

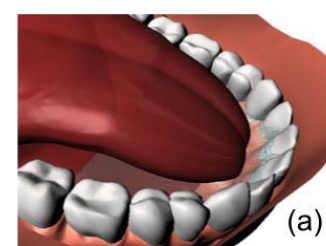

(a)

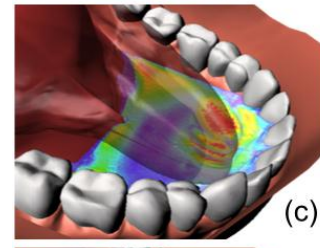

(c)

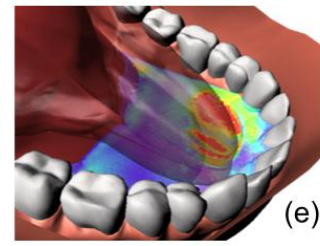

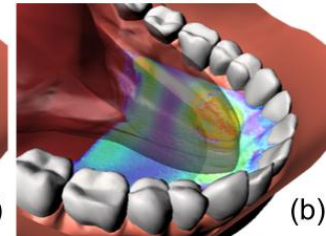

(b)

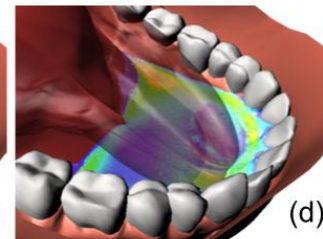

(e)

0.05

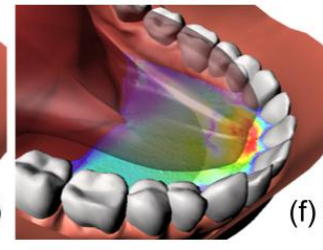

0.1

Velocity $(\mathrm{m} / \mathrm{s})$
Figure 2. Saliva motion in the anterior oral cavity induced by tongue movement and inertial forces with fluid coloured by velocity.

Ingestion and mastication begin the digestive process, which is critical to sustaining human life. Study of the processes that lead to the breakdown of food and release of flavor can assist in the understanding of oral digestive dysfunction and in the relationship between food structure and the sensory enjoyment of food. Experimental measurements of the products of chewing and the rates of transport of flavor components are often impractical (if not impossible) in vivo. Modeling of these phenomena is sparse (Dejak et al. 2003; de Loubens et al. 2011), limited by complexities such as large deformations (and fracturing) of solid materials combined with mixing in fluids. The Lagrangian SPH method enables straightforward representation of these very large deformations, microscale damage and subsequent fracturing of solids, interaction with complex moving and deforming boundaries, and chemical diffusion. SPH is therefore especially suited to modelling of food breakdown and flavour release in the mouth. Four examples of a coupled SPH-biomechanical model to processes of ingestion and mastication are presented.

Accurate modeling of saliva flow is important for predicting chewing and swallowing. The interaction between saliva and complex, deforming boundaries (the tongue) is shown in Figure 2. The tongue is represented by a deforming surface mesh. The motion of the tongue was specified so that it enters a pool of saliva in the anterior oral cavity (Figure 2b) and moves right (Figure 2c) to left (Figure 2d) and back again (Figure 2e). The tongue then exits the fluid (Figure 2f). Ripples are induced in the fluid from tongue entry and subsequent motion. High velocities are induced under (and ahead of) the tongue as it stirs the fluid. This demonstrates that interaction of the fluid with the complex moving and deforming geometries of the soft palate, gums and teeth can be modeled in a straightforward manner.

Biting reduces the size of ingested foodstuffs to suit chewing and swallowing. The magnitude of biting force and the resultant shape of the bitten food depend on the stiffness of the food, its internal structure and its propensity for either plastic deformation or brittle fracture. The inherent variability of the mechanical and breakage properties of these foodstuffs strongly influences the real and model outcomes. Figure 3 shows the large scale deformation and eventual separation of an example elastoplastic foodstuff by biting. Biting was simulated as constant speed rotational motion of the lower jaw until the incisors make contact with each other. Initially stress is localised to the regions near to the teeth (shape B). However, the jelly bean material between the teeth soon yields and plastic flow of the bean occurs (shape). The teeth eventually squeeze the bean into two discrete pieces as they make contact (shape D). The variation of the jaw force throughout the biting process is shown in Figure 3b. A major source of variability in such processes relates to the differences 
(a)
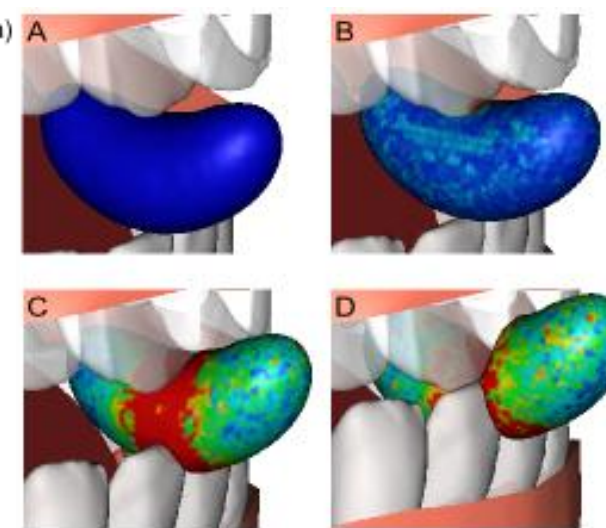

(b)

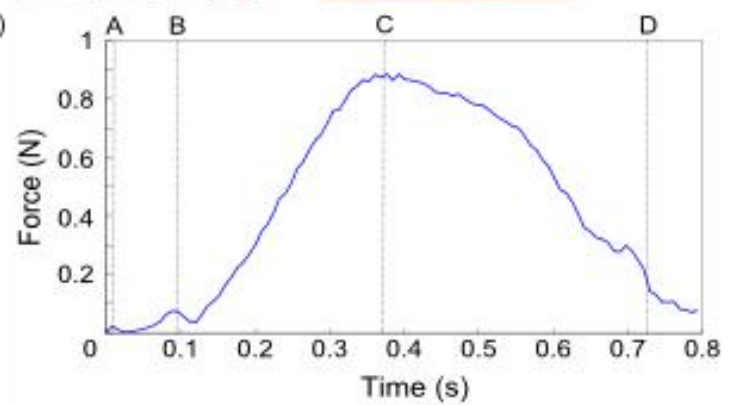

Figure 3. Variation of (a) the shape of a jelly bean, and (b) the vertical component of jaw force during biting by the incisors. The jelly bean is coloured by von Mises stress at times A, B, C and D.

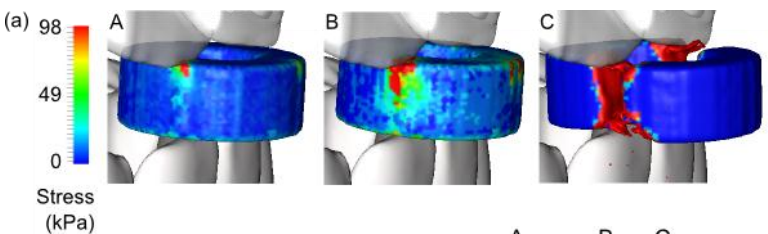

(b)

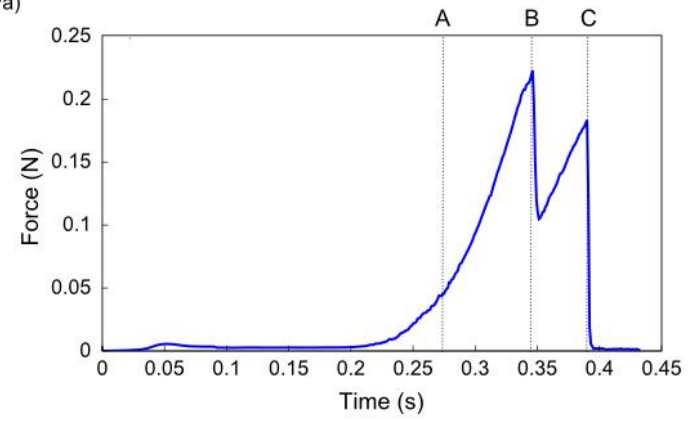

Figure 4. Variation of (a) the shape of a brittle hoopshaped candy, and (b) the vertical component of jaw force during biting. The candy is coloured by von Mises stress at times A, B and C. in teeth and mouth shape and particularly to the large variations in the motion of the tongue (responsible for holding food in place and transporting fragments) and in the motion of the jaw. Little quantitative information is currently available to characterise such behaviours, but the accuracy of the motions can have very strong effects on the model predictions. Customisation of the model to specific individuals is entirely limited by the difficulties in obtaining good quality detailed mouth and jaw motion information.

Fracturing of a brittle hoop shaped candy by the incisors is shown in Figure 4. Microscale cracking initiates first at the contact points with the teeth (candy). Jaw load continues to rise until cracks accumulate into fracture on the far side of the candy (shape B). The jaw load drops immediately after fracture because the bulk stiffness of the candy has been reduced markedly. Jaw load rises as the teeth further bite into the candy until cracks on the near side also accumulate into large scale fracture. The candy separates into two large and many small pieces and the jaw load quickly declines to zero (shape C). Unlike the elastoplastic jelly bean, the brittle candy is broken before the teeth make contact. The jaw load declines very quickly once fracture has occurred (in less than 10 ms, see Figure $4 b$ ). This is fast compared to biting an elastoplastic material where the jaw load declines more slowly (over $400 \mathrm{~ms}$ ).

Chewing follows biting as the ingested foodstuff is broken down further and mixed with saliva in preparation for swallowing. Squashing of an elastoplastic model jelly baby by the molars is shown in Figure 5. Jaw motion is represented by periodic translations in all three directions. Jaw load is zero until both the top and bottom molars are in contact with the jelly baby (shape). The peak in jaw load corresponds to the timing of the maximum vertical displacement of the lower jaw. The material is deformed significantly as the approaching molars squeeze the jelly baby medially and laterally to the teeth contact surfaces (shape B). As the lower jaw displaces downwards, contact is lost between the jelly baby and the top teeth (shape $\mathrm{C}$ ). The variation of the jaw force is shown in Figure 5b. Due to large scale yielding of the jelly baby, little elastic recovery is seen after loading is removed.

These applications show the applicability of the coupled SPH-biomechanical model to study oral food breakdown. Advantages of SPH over grid based methods for these applications are numerous. Determination of the free surface of the saliva in the first application is difficult using grid based models and the deforming mesh of the tongue presents further complications. The fracturing of the brittle solid and the flow of the elastoplastic solids in the biting and chewing applications require re-meshing and interpolation of state variables, which can be difficult for very large deformations. Future models will incorporate mixing of broken down foodstuffs with saliva, flavour diffusion and swallowing. Significant study is required to understand the effect of variations in chewing and tongue motions, both between cycles for the same person and for variation between people. 


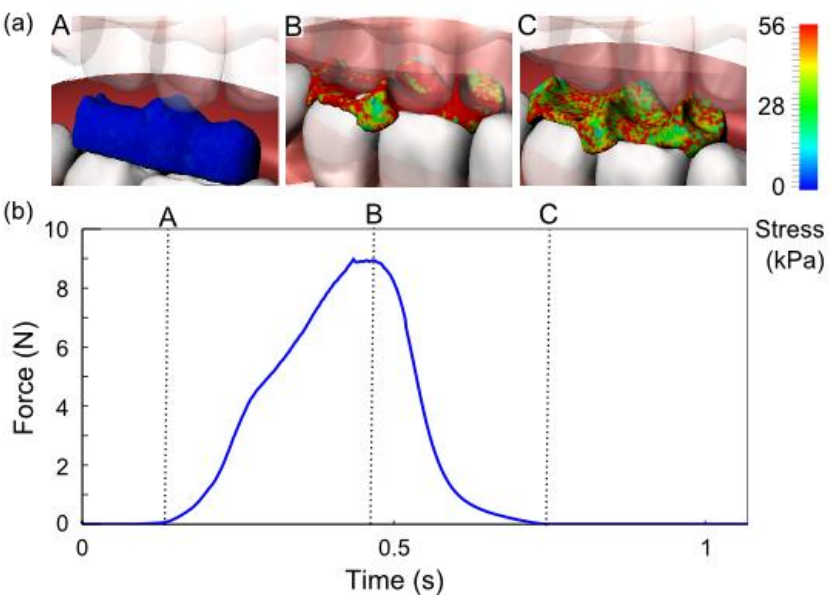

Figure 5. Variation of (a) the shape during chewing of a jelly baby, and (b) the magnitude of jaw force. The deformed shape of the jelly baby, coloured by von Mises stress, is displayed at three times A, B and C.

\section{SPORTS}

Understanding of human swimming techniques requires experiments that can be performed in a controllable and repeatable manner. Computational Fluid Dynamics (CFD) based experimentation satisfies these requirements and provides full details of the fluid flow around the swimmer. Using this information the critical flow structures can be identified, forces can be quantified and the work exerted by the swimmer can be calculated. Not only can different swimming techniques be studied and compared in this way, but other factors critical to performance can be investigated including the effects of morphology, strength and flexibility.

Human swimming offers a unique combination of modelling challenges including high Reynolds number flows; complex free surface motion including splashing and entrainment of gas; and interaction with a rapidly deforming biomechanical model of the swimmer. SPH is well suited to these demanding requirements due to it mesh-less nature which allows the distribution of particles to conform to both the swimmer geometry and to the fluid free surface. The recent study of submerged dolphin kick swimming (Cohen et al., 2011) demonstrated the emergence of SPH as a tool for studying swimming stroke technique.

In order to study swimming at the elite level, the biomechanical model must include all the subtle details of the swimmer technique and body positioning. Marker based motion-capture techniques have large inherent uncertainties that make this requirement difficult to satisfy. The present method used for the kinematics digitisation of a human swimmer is a manual process that is highly accurate and well suited for subsequent
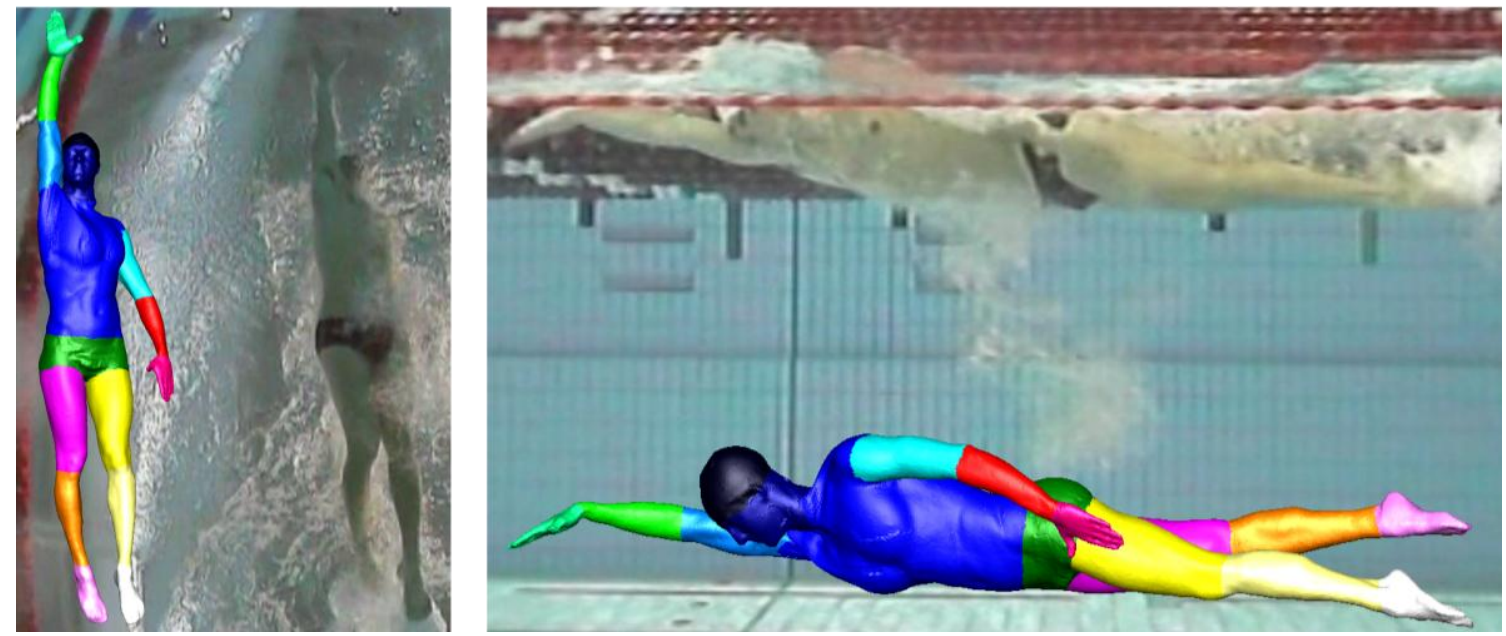

Figure 6. Articulated swimmer model and the corresponding frames of multi-angle footage, (left) bottom-up view; and (right) side-on view.

use in fluid dynamics simulations. The biomechanical model for a human swimmer is created by firstly taking a laser body scan of a swimmer in a single anatomical pose. This is then "rigged" to an underlying skeletal structure using the commercial animation package Maya (Autodesk Inc, San Rafael, CA, USA). Rotations of the skeleton joints enable the swimmer model to attain new poses. Video footage of the athlete swimming in a pool is taken using multiple cameras that track alongside and directly underneath the swimmer. This keeps the swimmer fixed within the frame of each camera and provides enough information to determine the three-dimensional kinematics of the swimmer. To animate the rigged swimmer model, it is placed alongside the footage and articulated to match each frame. An example of such side-by-side animation of the biomechanical model is shown in Figure 6, which has a male swimmer performing freestyle. Despite 
issues of bubbles, refraction and lens distortion, the footage is rich in information and enables highly accurate kinematics to be generated.

Simulation of a virtual male freestyle swimmer in a tank is shown in Figure 7a. This highlights the fluid free surface, from the splashes being generated by the arms and legs to the bow waves being generated by the swimmer motion. Since the swimmer motion is dynamic, his translational and rotational speeds are determined by the fluid mechanical forces and torques that the water exerts on him. By adjusting the base stroke technique and re-running the swimming simulations the implications of changing from one stroke to another can be determined. This can explain why one kind of stroke optimisation may work for some individuals but not others.

Details of the flow on the sagittal $(z$ $=0$ ) plane around the swimmer are shown in Figure $7 \mathrm{~b}$. The plane is coloured by velocity magnitude, highlighting the high speed fluid in the vicinity of the swimmer and being generated by the feet. Also shown are the in-plane streamlines in the reference frame of the moving swimmer. The flow remains attached along the torso but separates below the waist. Figure 7c displays the sagittal pane coloured by spanwise vorticity. The shear layers along the torso are clearly visible and the complex wake behind the swimmer is also evident. CFD visualisations like these can be used to identify important flow bifurcations that occur as the swimming technique is varied.

Another related sport which can benefit from CFD is diving. The same multi-angle marker-less motion capture technique is well suited to the free-fall portion of dives. An example of an SPH simulation of a vertical dive by a female diver is shown in Figure 8. Once the biomechanical diver model hits the water, the interaction causes it to slow and the water to produce a splash. Figure 8 shows both the development of the splash, the vorticity on the $\mathrm{z}=0$ plane and the dynamic motion of the diver. At time t0 the diver begins to enter the water vertically at high speed and is fully submerged $0.17 \mathrm{~s}$ later. This entry causes a large void to
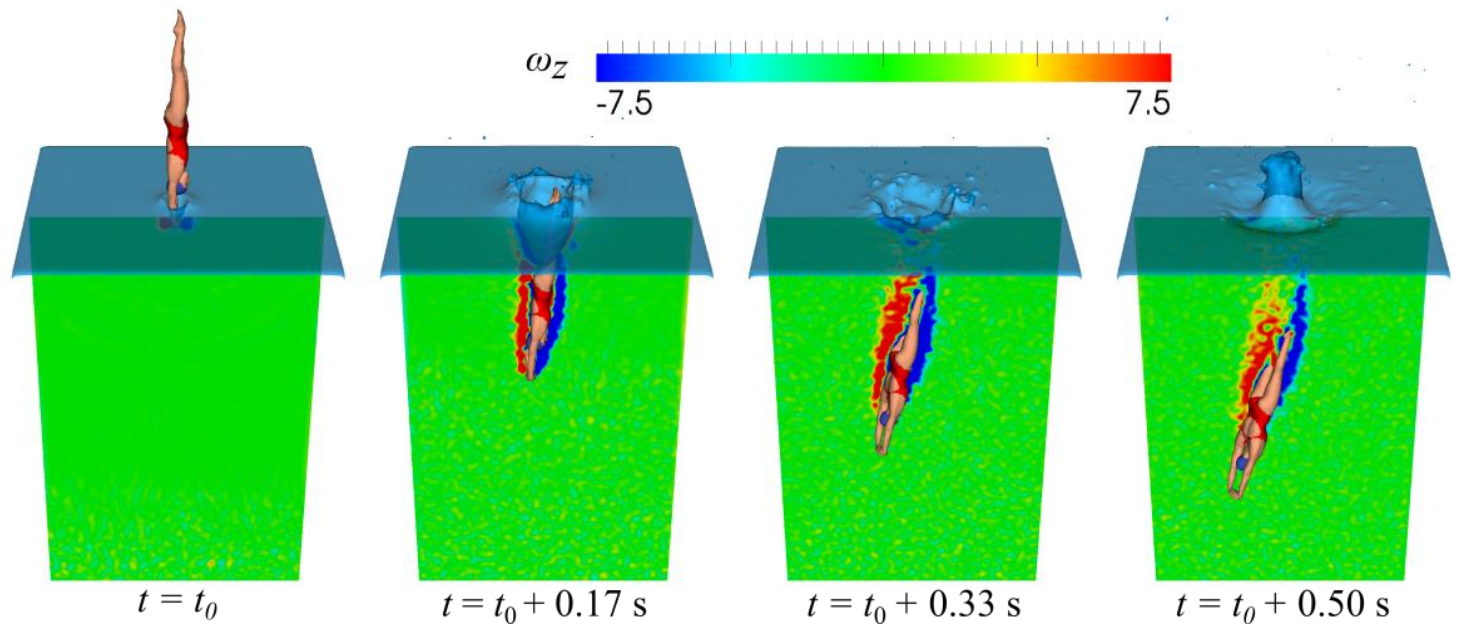

Figure 8. Sequence of free-surface and $z=0$ plane visualisation during a female dive. 
form in the water behind the descending diver. A further 0.16 seconds later the diver has slowed down considerably and rotated off the vertical. Strong shear layers are visible around the body and the surrounding water has rushed to fill the void. By time $t 0+0.50 \mathrm{~s}$ the diver has slowed down further and a large splash is rising from the pool. Such modelling provides an ideal environment for studying different entry speeds, entry angles and body positioning because the resultant motion and splashing can be objectively compared.

For both sports, much of the variability that needs to be coped with in the modelling relates to variation in the athletes' body deformation between repetitions, between training and competition, changes in their technique with injury and fatigue and the very large variations that occur between athletes. There can also be large variations in the conditions in which they perform (e.g. large waves from other competing swimmers).

\section{BIOMEDICAL}

The operation of biomedical systems often involves interactions between multi-phase fluids (blood, urine, faeces) and surrounding soft tissues and muscle embedded in a dynamic biomechanical system that is the human body. Two examples of biomedical applications where we have applied SPH are presented below.

\subsection{Cardiac Flow Assistance (Axial Flow Pump)}

Ventricular assist devices (VADs) are used as a temporary bridge-to-transplant for patients with congestive heart failure, or as flow assistance for instances of heart disease where ventricular function is weakened. CFD has been used in the heart pump industry for more than a decade, and is a well-established tool for pump optimisation. To date, this has only involved grid-based methods and almost entirely focused on studying steady flow conditions. However, models of transient flow are necessary for designers to understand the velocity and pressure fluctuations occurring in a pump under unsteady flow conditions as would occur in a clinical, in vivo setting. In addition, axial flow pump designs use very high rotor speeds (up to 10,000 rpm) which can lead to significant haemolysis (red blood cell damage).

SPH offers some advantages over grid-based methods for pump applications in medicine. There is no need for slip meshes to manage moving machine components, nor the potential mass and energy losses associated with flow from moving meshes to stationary ones. In SPH, boundary geometries of almost arbitrary complexity may be included such as intricate pump rotor geometry. SPH particles can also carry fluid history easily, enabling inclusion of strain-based models for blood cell damage. Finally, SPH enables rule-based modification of particles, meaning that one could also potentially model the growth of thrombi inside the pump. Predictions of cell damage and clotting inside the pump are important for understanding the useful life of a pump and pose significant challenges for grid-based methods.
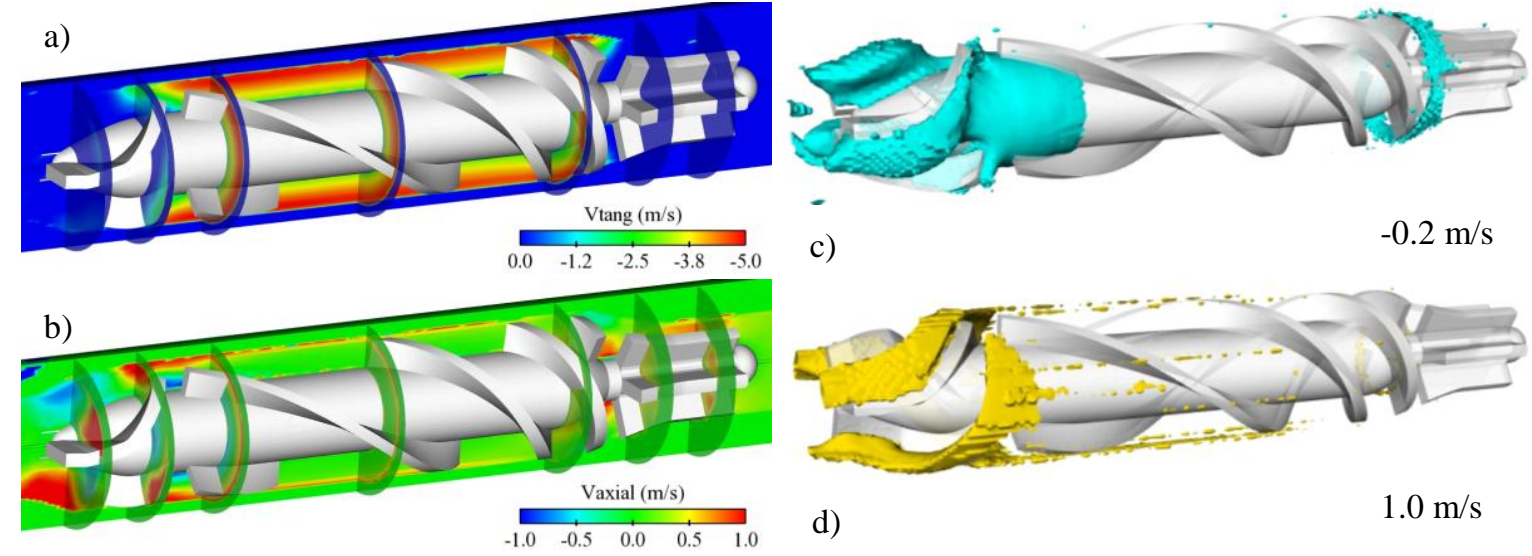

Figure 9. Average a) tangential and b) axial flow velocities within the pump housing. Isosurfaces of axial velocity $\left(\mathrm{V}_{\mathrm{z}}\right)$ demonstrating retrograde flow in the pump for $\left.\mathrm{c}\right)-0.2 \mathrm{~m} / \mathrm{s}$, and d) $1.0 \mathrm{~m} / \mathrm{s}$.

Sinnott and Cleary (2010) have used SPH to model flow through an axial flow pump (shown in Figure 9). Figure $9 \mathrm{a}$ and $\mathrm{b}$ show the tangential and axial velocity flow field. Here, the impeller rotation draws blood through the pump from right-to-left and converts the flow from linear motion (by the stationary inducer blades) to rotational motion (by the rotating impeller blades) and back to linear (in the stationary diffuser) with a subsequent rise in pressure head at the outlet of the pump. This is intended to approximate real cardiac pressures. Isosurfaces of axial velocity are shown in Figure 9c (reverse flow) and Figure 9d (forwards flow) and demonstrate recirculation inside the pump. Regions of recirculation are of concern for pump designers as 
they can trap blood inside the pump increasing the exposure time for red blood cells to significant levels of shear and increasing the potential for blood damage.

\subsection{Gastro-intestinal Transport}

Controlled propulsion and mixing of content along the digestive tract is essential for a normal life. This is achieved by a rich assortment of motor patterns that ensure that movements and propulsion are appropriate for the breakdown of food, absorption of nutrients and excretion of waste. These movements (motility) are due to coordinated contractions and relaxations of circular and longitudinal smooth muscle layers. Computational modelling of gastrointestinal systems has the potential to help understand complex relationships between flow and pressure for functional disorders where intestinal transport is abnormal. However predicting the transient fluid-structure interactions which lead to large wall deformations poses significant challenges for grid-based methods.

The mesh free nature of SPH means that large deformations can be modelled without requiring expensive and diffusive re-meshing. Coupling of fluid/solid motion and wall deformation is captured naturally. Here, we use SPH particles to model both the faecal content and the intestinal wall. Viscoelastic elements are constructed between each pair of wall particles to allow the wall to flex in response to applied muscular contractions and fluid pressures from the faecal content. This is a powerful and effective model for an active boundary arising from the tightening or relaxing of circular muscle in the intestinal wall. The key advantage of this model is that the instantaneous shape of the wall is a direct prediction from the fluid-structure interaction between faecal content and muscular control of the boundary. This is in contrast with earlier models (e.g. Pal et al. 2004) where the wall deformations were specified as inputs to the model thereby introducing significant uncertainty about the predictive capability of such models.

Travelling peristaltic waves are generated in the intestinal wall and travel in the antegrade direction. The wave consists of contraction (ascending excitation) and relaxation (descending inhibition, DI) components that modify the instantaneous wall tensions in each longitudinal slice by controlling the natural lengths of the elastic wall elements and thereby produces the required degree of contraction or relaxation. Complex propagating sequences in the colon can easily be represented by series of these waves.

Figure 10 shows an example of peristaltic transport in the colon where the wave is travelling from left-toright. The downstream muscular relaxation (DI) was shown to be important for both transport and mixing by Sinnott et al. (2011). A high pressure zone propagates immediately in front of the contraction such that fluid wants to expand sideways. The addition of DI allows the walls to expand. Consequently there is a reduction in luminal and intra-luminal pressures inside the region of DI which generates positive thrust for the fluid volume contained inside the dilation region. This then travels forwards at the propagation speed of the contraction. A recirculation vortex forms inside the DI (shown by the velocity field in Figure 10) that influences the wall shape by controlling the pressure distribution, but the wall shape also influences the size and shape of the vortex since they are coupled. The largest source of variability here are the details of the peristaltic waves, which vary in timing, magnitude, degree of occlusion, location and speed. The waves can also interact due to overlapping of wave effects due to their long relaxation times of the wall-fluid system.

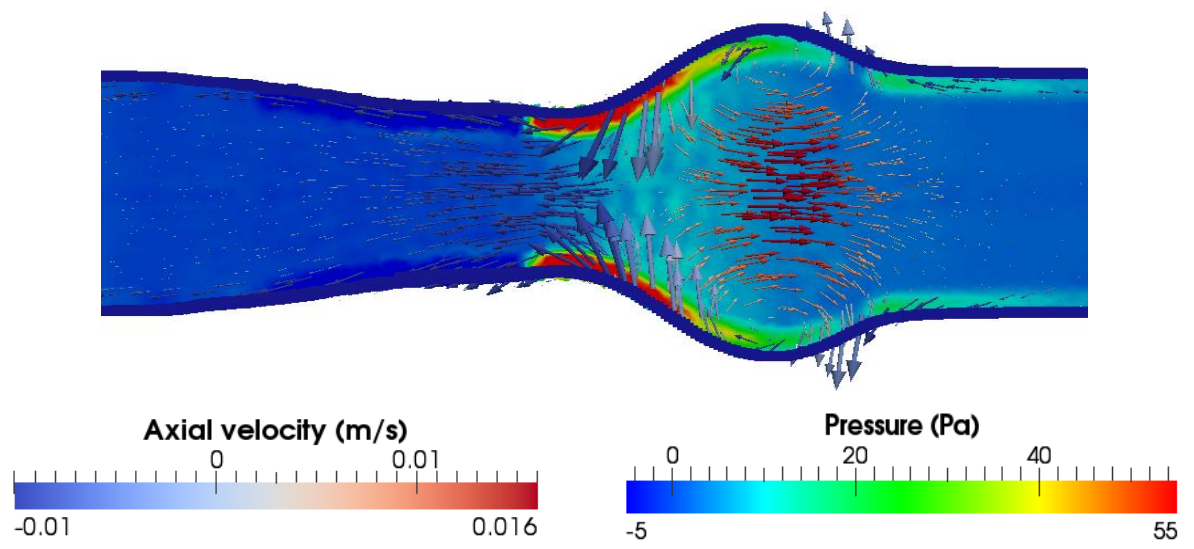

Figure 10. Vertical slice through the centreline of the colon with faecal content (fluid) colour-shaded from blue to red by pressure. Velocity arrows are coloured by longitudinal speed for antegrade (red) and retrograde (blue) flow and the arrow length represents longitudinal speed. 


\section{GEOPHYSICAL}

Extreme geophysical flows such as tsunamis and dam breaks occur on large scales, have complex free surfaces that interact with complex terrain and usually have catastrophic consequences for life and property. Predicting the outcomes of such events is desirable for hazard mitigation and disaster management. The free surface motion, interactions with complex terrain and large spatial areas which characterize geophysical flows make experimental and physical measurement almost impossible. As a result of this, much focus has been placed on numerical modeling of these events. Modelling is a key element of understanding risk, planning for disaster and developing mitigation strategies. The simulation tools are now advanced enough to make comprehensive predictions of the consequences for specific scenarios. The utility of such modelling is now entirely limited by the ability to understand and characterize the space of possible disaster scenarios. When will the events occur? How big will they be? What is the possible range of variations? Combining modelling of the scenario space is now an important area of work for such disaster and risk modelling.

\subsection{Dam Breaks}

Multiple failure scenarios of the Geheyan dam located in central China were simulated to understand the effect the failure scenario has on downstream inundation. Water velocities and downstream flood levels are shown in Figure 11 for two failure scenarios, a) complete instant collapse, and b) collapse of just the upper dam wall section due to overtopping of the dam. The water is shaded by velocity with blue being low, green being moderate speed $(12.5 \mathrm{~m} / \mathrm{s})$ and red being high speed $(25 \mathrm{~m} / \mathrm{s})$.

In the total collapse scenario, the leading edge of the flood has nearly reached the post office (location 5) and key locations 1-4 have significant flooding. Two large hydraulic jumps occur immediately downstream of the dam wall and there is also significant upstream flow into a tributary $1.5 \mathrm{~km}$ downstream. In the overtopping collapse scenario, the leading edge of the flood has not travelled as far. The first two downstream hydraulic jumps have been suppressed by the presence of the large dam fragment that broke from the dam wall. Key locations 2 and 4 have not been flooded yet and there is much less flow up the tributary. In general, the flooding is less widespread and the water velocities are much lower than for total collapse. The large differences in inundation indicate the importance of the failure modes.

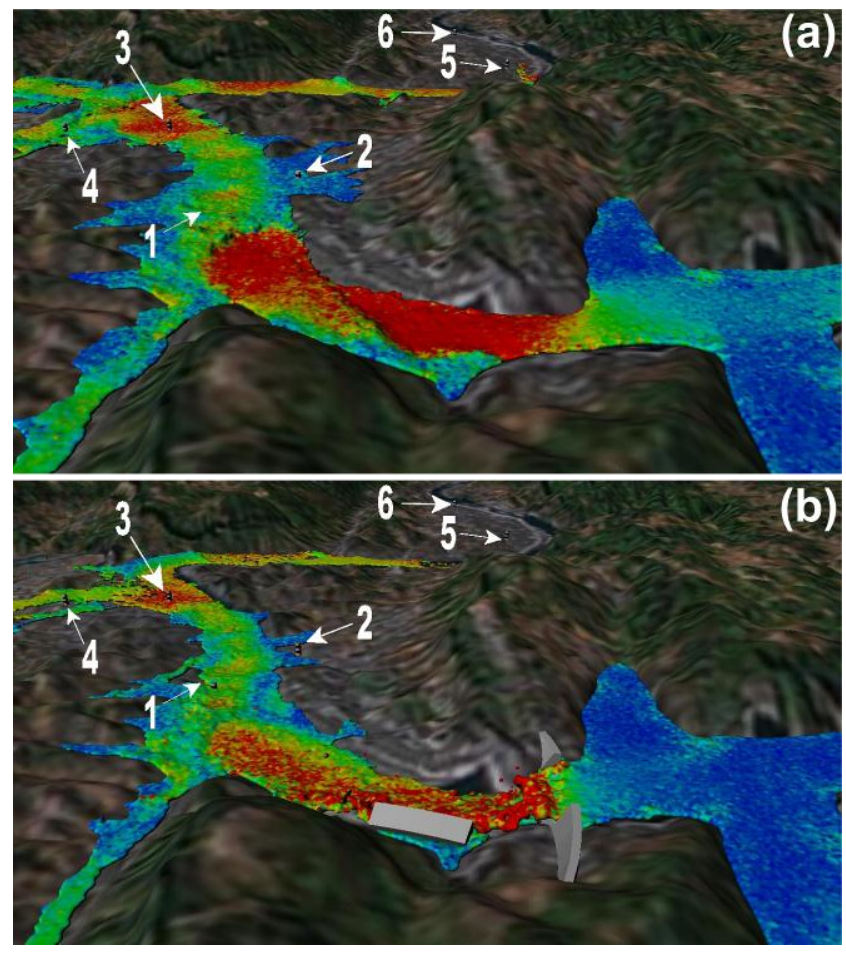

Figure 11. Inundation downstream of Geheyan dam after five minutes, for (a) instantaneous total collapse, and (b) overtopping collapse. Key locations of a fuel station (1), pre-school (2), mobile phone tower (3), primary school (4), post office (5) and bank (6) are indicated.

The interaction of the flow with the buildings generates complicated three dimensional flow structures that affect the direction and magnitude of the flooding within urban areas. Accurate prediction of flooding in such areas allows damage assessments based on likely flooding scenarios. Inundation of a town $4 \mathrm{~km}$ downstream of Geheyan dam wall was simulated and is shown in Figure 12. The town consists of 40 buildings and a bridge. Figure $12 \mathrm{~b}$ shows the flood waters arriving at the town after $2 \mathrm{~min}$. The large warehouse closest to the dam wall obstructs the flow, causing it to separate into two streams. It is likely that this structure would collapse due to the forces exerted on it. The flooding after 3 min (Figure 12c) shows that most of the town has been inundated. The large warehouse is now completely underwater and the buildings closest to the dam wall reduce the velocity of the water in the town to less than $12.5 \mathrm{~m} / \mathrm{s}$. After $6 \mathrm{~min}$ almost all the buildings in the township are completely inundated (Figure 12d). Ensemble weighting of such predictions can be used for risk assessment, planning, mitigation and pricing of insurance and reinsurance. 


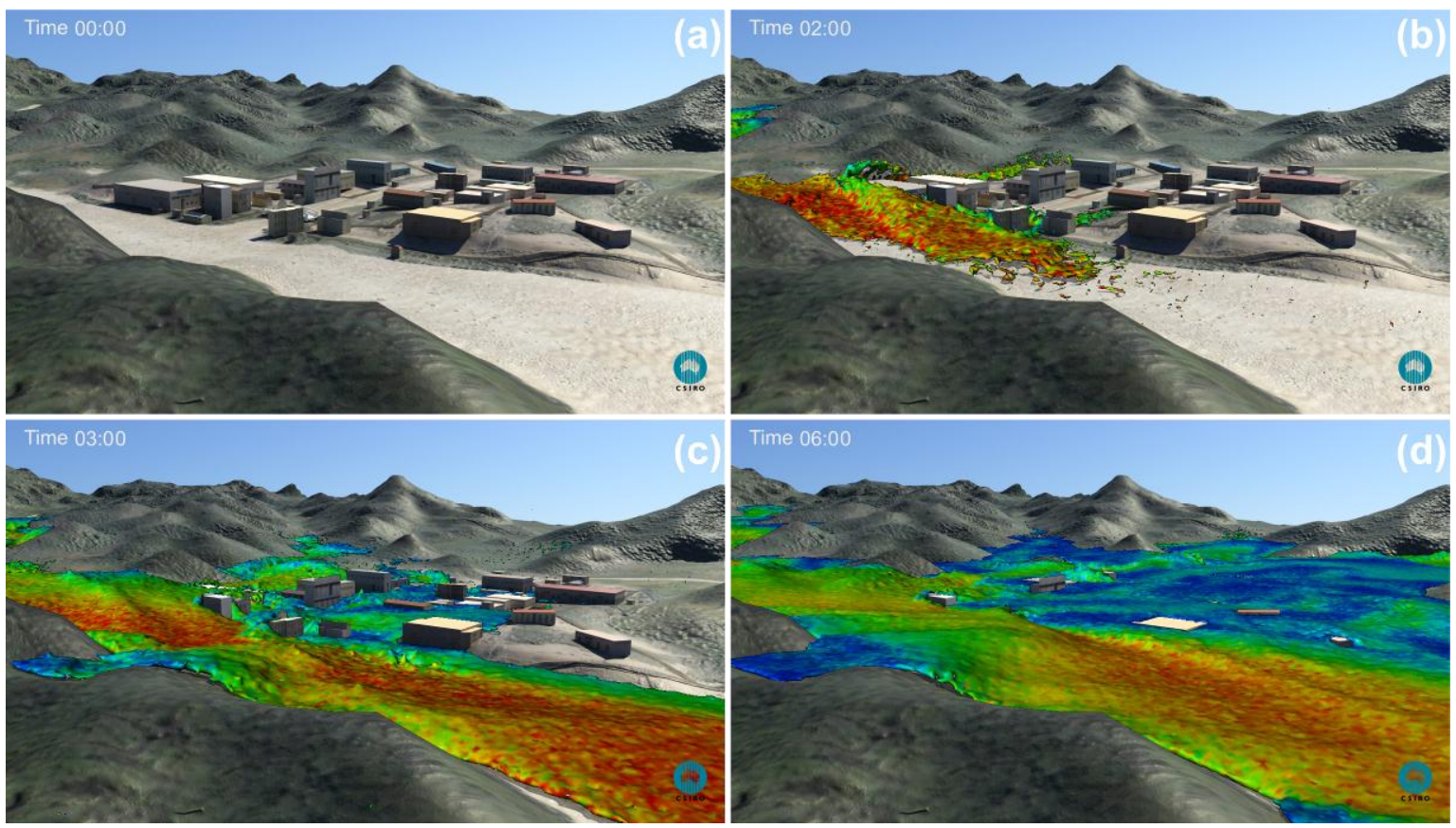

Figure 12Inundation of an urban area $4 \mathrm{~km}$ downstream of the Geheyan dam wall, modelled using SPH at (a) 0 min, (b) 2 min, (c) 3 min and (d) 6 min.

\subsection{Tsunamis}

The impact of tsunamis on coastal areas causes devastating property damage and potentially significant loss of life. The extent of the damage depends on the strength and angle of incidence of the tsunami wave. The local bathymetry of the ocean floor and the topography of the coastal terrain also have a major influence on the resulting damage. These can be investigated using numerical models. SPH has several advantages over traditional mesh based techniques for simulating tsunami inundation. These include resolution of the wave structure, the three dimensional nature of the tsunami resulting from non-uniform bathymetry and coastal topography and interaction with complex natural or human-made structures.
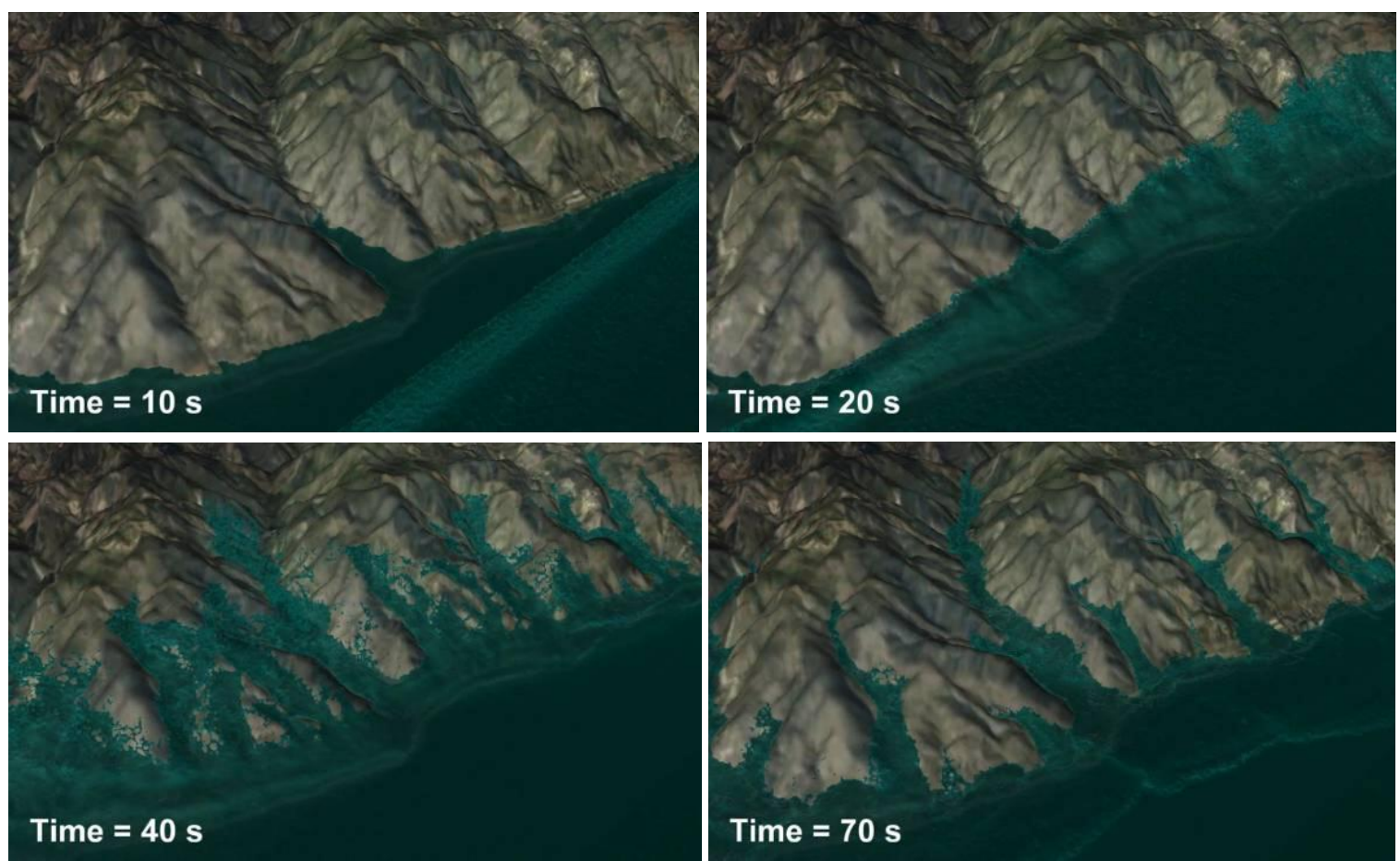

Figure 13. A $50 \mathrm{~m}$ high tsunami moving at $15 \mathrm{~m} / \mathrm{s}$ inundating parts of northern Californian coastline. 
Figure 13 shows a $50 \mathrm{~m}$ high tsunami wave moving at $15 \mathrm{~m} / \mathrm{s}$ inundating parts of the northern Californian coastline. At $10 \mathrm{~s}$, the wave is approaching the coastline from the southwest at a $30^{\circ}$ angle, with the southern sections beginning to be inundated. At $20 \mathrm{~s}$, the wave has now impacted the entire length of the coastline. The water has started moving inland in the southern section. By $40 \mathrm{~s}$, the water has moved $1.5 \mathrm{~km}$ inland. At $70 \mathrm{~s}$, the water has started receding from the southern sections but is still moving further inland along the central and northern sections. The water has inundated around $2 \mathrm{~km}$ inland along the central valley and is now approaching its maximum levels. The detailed predictions of such a deterministic model can be used within a risk frame work to determine the consequences for the different scenarios considered.

\section{CONCLUSIONS}

Particle based computational methods have a range of strong advantages over traditional grid based continuum methods. These include:

- the ability to include particle level information in collision dominated particle flows

- ability to resolve complex fluid free surfaces including splashing and fragmentation

- ability to predict very large deformations, including fracture and interaction with moving objects

- ability to track material history and use this in the flow modelling.

These have been demonstrated in a broad range of computationally demanding applications including comminution, biomedical, geophysical extreme flow events (risk/disaster modelling), eating of food by humans and elite water based sports. In all cases, there are significant sources of variability that need to be taken into account in the modelling. For human based models the major sources of variability are the nature of the motions of the human (both internal and external). For the disaster modelling, the variability is mainly reflected in the range and nature of scenarios that need to be considered in building risk frameworks in which these models can predict specific consequences. Finally, in all the applications there is commonly uncertainty about material properties and the details of the initial conditions whose affects need to be understood.

\section{ACKNOWLEDGMENTS}

The authors acknowledge the financial and material contributions of the Aquatics Testing, Training and Research Unit (ATTRU) at the Australian Institute of Sport (AIS) to the human swimming research.

\section{REFERENCES}

Campbell, C. S. (1990). Rapid granular flows. Annual Review Fluid Mechanics 22, 57-92.

Cleary, P. W. (2004). Large scale industrial DEM modelling. Engineering Computations 21, 169-204.

Cleary, P. W. (2009). Industrial particle flow modelling using DEM. Engineering Computations 26, 698-743.

Cleary, P. W., Prakash, M., Ha, J., Stokes, N., and Scott, C. (2007). Smooth Particle Hydrodynamics: Status and future potential, Progress in Computational Fluid Dynamics, 7, 70-90.

Cohen, R. C. Z., Cleary, P. W., and Mason, B. R. (2011). Simulations of dolphin kick swimming using smoothed particle hydrodynamics.Human Movement Science. doi:10.1016/j.humov.2011.06.008.

Cundall, P. A., and Strack, O. D. L. (1979). A discrete numerical model for granular assemblies. Geotechnique. 29, 47-65.

Dejak, B., Mlotkowski, A., and Romanowicz, M. (2003) Finite element analysis of stresses in molars during clenching and mastication. The Journal of Prosthetic Dentistry, 90(6), 591-597

de Loubens, C., Saint-Eve, A., Déléris I., Panouillé, M., Doyennette, M., Tréléa, I.C., and Souchon, I.. (2011) Mechanistic model to understand in vivo salt release and perception during the consumption of dairy gels. Journal of Agricultural and Food Chemistry, 59(6), 2534-2542.

Haff, P. K., and Werner, B. T. (1986). Computer simulation of the mechanical sorting of grains. Powder Technology. 48, 239.

Monaghan, J. J. (1994). Simulating free surface flows with SPH. J. Computational Physics 110, 399-406.

Pal, A., Indireshkumar, K., Schwizer, W., Abrahamsson, B., Fried, M., and Brasseur, J. (2004). Gastric flow and mixing studied using computer simulation, Proceedings Royal Society. London B, 271, 2587-2594.

Sinnott, M., Cleary, P.W., Arkwright, J., and Dinning, P. (2011). Investigating the relationships between peristaltic contraction and fluid transport in the human colon using Smoothed Particle Hydrodynamics. Computers in Biology and Medicine. Submitted in 2011.

Sinnott, M., and Cleary, P.W. (2010). Effect of rotor blade angle and clearance on blood flow through a nonpulsatile, axial, heart pump. Progress in Computational Fluid Dynamics, 10, 300-306.

Walton, O. R. (1994). Numerical simulation of inelastic frictional particle-particle interaction, Chapter 25 in: Particulate two-phase flow, ed. M. C. Roco, pp. 884-911, Boston: Butterworth-Heinemann. 